\title{
Adaptación transcultural del cuestionario ISSIQ-A para evaluar Impulsividad, Autolesión No Suicida e Ideación Suicida en adolescentes mexicanos
}

\section{Transcultural adaptation of the Impulse, Self-harm and Suicide Ideation Questionnaire for Adolescents (ISSIQ-A) in a Mexican population}

\author{
Yolanda Chávez-Flores \\ Centro de Investigación en Riesgos y Calidad de Vida, Centro Universitario del Sur, \\ Universidad de Guadalajara. Cd. Guzmán. Jalisco, México \\ Carlos Hidalgo-Rasmussen \\ Centro de Investigación en Riesgos y Calidad de Vida, Centro Universitario del Sur, \\ Universidad de Guadalajara. Cd. Guzmán. Jalisco, México \\ Centro de Estudios Avanzados, Universidad de Playa Ancha, Valparaíso, Chile. \\ Fabiola González-Betanzos \\ Facultad de Psicología. Universidad Michoacana de San Nicolás de Hidalgo, México
}

Recibido (22 de enero de 2018) Aceptado (28 de mayo de 2018)

\begin{abstract}
Resumen
Se describe el proceso de adaptación transcultural del ISSIQ-A en adolescentes mexicanos entre 11 y 19 años de edad. Se realizaron dos traducciones y una retrotraducción verificando la equivalencia semántica y conceptual con la versión original en inglés. Se realizaron entrevistas cognitivas con la población objetivo y se comprobó la comprensión de los ítems, instrucciones y categorías de respuesta. La fiabilidad fue alta para el instrumento completo $(\alpha=.93)$ y moderada en la mayoría de las dimensiones $\left(\alpha_{\min }=.67\right.$ a $\alpha_{\max }=$.94). La reproducibilidad evaluada a una semana fue moderada. Tanto el efecto piso techo como la validez convergente y divergente fue adecuada. Mediante análisis factorial confirmatorio se comprobó el modelo de cuatro factores y dos subescalas. Los resultados indican que la versión mexicana del ISSIQ-A es un instrumento válido y fiable para identificar y evaluar la autolesión no suicida y variables asociadas en adolescentes mexicanos.

Palabras clave: Conducta autodestructiva, adolescente, encuestas y cuestionarios, comparación transcultural, psicometría.
\end{abstract}

Correspondencia: Carlos Hidalgo-Rasmussen. Centro de Investigación en Riesgos y Calidad de Vida. Universidad de Guadalajara. Av. Enrique Arreola Silva 883 Col. Centro, Cd. Guzmán, Jalisco, México. CP 49000. Correo electrónico carlos.hidalgo@academicos.udg.mx, chidalgor@outlook.com Agradecimientos: Agradecemos a Carolina da Motta coautora de la versión original del ISSIQ-A por su colaboración revisando la adaptación lingüística del instrumento. 


\begin{abstract}
This study described the transcultural adaptation process of the ISSIQ-A in a Mexican population of adolescents between 11 to 19 years old. Two translations and a back-translation were carried out, verifying the semantic and conceptual equivalence with the original version in English. Cognitive interviews were conducted with the target population and the understanding of the items, instructions and response categories were verified. The reliability was high for the complete instrument $(\alpha=.93)$ and moderate for most of the dimensions $\left(\alpha_{\min }=.67\right.$ to $\left.\alpha_{\max }=.94\right)$. The reproducibility evaluated at one week was moderate. Both the floor and ceiling effect and the convergent and divergent validity were adequate. Through confirmatory factor analysis, the four-factor model and two subscales were confirmed. The results indicate that the Mexican version of the ISSIQ-A is a valid and reliable instrument to identify and evaluate non-suicidal self-harm and associated variables in Mexican adolescents.
\end{abstract}

Keywords: Self-injurious behavior, adolescent, surveys and questionnaires, cross-cultural comparison, psychometrics.

\section{Introducción}

La autolesión no suicida (NsSI por sus siglas en inglés) es la destrucción directa y deliberada de tejido corporal en ausencia de intención suicida (Nock, 2010). Los métodos de NSSI más frecuentes incluyen cortarse la piel, arañarse, golpearse, interferir con la cicatrización de heridas, morderse, quemarse y enterrarse agujas (Saraff \& Pepper, 2014). En el periodo 2005-2011, la prevalencia internacional de conductas de NSSI en adolescentes se estimó en 18\% (Muehlenkamp, Claes, Havertape \& Plener, 2012) y la edad de inicio entre los 12 y 16 años (Nock \& Prinstein, 2004; Muehlenkamp \& Gutierrez, 2007). Existen diversos instrumentos desarrollados para evaluar la conducta de NSSI en adolescentes (Washburn, Juzwin, Styer \& Aldridge, 2010; Manca, Presaghi \& Cerutti, 2014; Vrouva, Fonagy, Fearon \& Roussow, 2010), sin embargo, los que se encuentran disponibles en español son menos (Calvete, Orue, Aizpuru \& Brotherton, 2015) y específicamente en México existe uno para evaluar este tipo de conducta, pero en adultos jóvenes (Castro et al., 2016). Actualmente existe un vacío de instrumentos diseñados para la población adolescente, que es el principal grupo de edad donde la conducta de Nssi se presenta (Hawton \& Van Heeringen, 2008; Klonsky, 2011) por lo tanto resulta necesario contar con instrumentos de evaluación confiables y válidos, que puedan utilizarse en el contexto mexicano, tanto en la práctica clínica como en investigación.

El Cuestionario Impulsividad, Autolesión No Suicida e Ideación Suicida para adolescentes (ISSIQ-A por sus siglas en inglés del Impulse, Self-harm and Suicide Ideation Questionnaire for Adolescents; Carvalho et al., 2015) es un instrumento de autoreporte que cuenta con evidencia de validez y confiabilidad. Su objetivo es evaluar las conductas y funciones de la NSSI, además de la impulsividad, los comportamientos de riesgo y la ideación suicida, ya que diversos estudios han mostrado su relación con las conductas de autolesión (Hamza, Willoughby \& Heffer, 2015; Duggan \& Heath, 2013; Tørmoen, Rossow, Larsson \& Mehlum, 2013). Fue desarrollado en Portugal con una muestra de 1722 estudiantes adolescentes entre los 14 y 21 años de edad $(M=16.5, S D=1.30)$. Consta de 56 ítems y las opciones de respuesta consisten en una escala Likert de cuatro puntos que van del $0=$ Nunca me ocurre al $3=$ Me ocurre todo el tiempo. Para garantizar su validez de contenido, se revisó a profundidad la literatura, previo a la construcción y selección de los ítems y se realizaron entrevistas cognitivas con la población meta para asegurar que tanto los ítems como las instrucciones fueran comprensibles. Se encuentra disponible en los idiomas portugués e inglés. Respecto a sus propiedades psicométricas, existe evidencia de su validez de constructo mediante un análisis factorial confirmatorio cuyos resultados mostraron un modelo de cuatro dimensiones con buen ajuste y fiabilidad: $X^{2}{ }_{(261)}=2133.025, p<$ .001 ; CFI $=0.901$; RMSEA $=0.065$, PCFI $=0.784$; $\mathrm{AIC}=$ 2311.025, Impulsividad ( 8 ítems, $\alpha=.77$ ), Autolesión No Suicida ( 8 ítems, $\alpha=.90$ ), Conductas de Riesgo (6 ítems, $\alpha$ $=.81$ ), Ideación Suicida ( 3 ítems, $\alpha=.82$ ) y dos subescalas con buenos índices de ajuste $X_{(425)}^{2}=767.041, p<.001$; CFI $=0.993 ; \mathrm{TLI}=0.992 ; \mathrm{RMSEA}=0.022$, Reforzamiento Automático (24 ítems, $\alpha=.93$ ) y Reforzamiento Social ( 7 ítems, $\alpha=.77)$. La consistencia interna del instrumento completo es de $\alpha=.86$. Además, se verificó la validez convergente a partir de las correlaciones positivas y negativas con otros instrumentos (Carvalho et al., 2015).

Aunque existen estudios con población comunitaria donde se muestra que las conductas de NSSI son frecuentes en los adolescentes en México (Albores-Gallo et al., 2014; Mayer, Morales, Figueroa \& Ulloa, 2016), hasta donde conocemos, no existe evidencia de instrumentos creados o 
adaptados en el contexto mexicano para evaluar la autolesión no suicida en adolescentes, por lo que el propósito de este estudio fue adaptar transculturalmente el cuestionario ISSIQ-A en adolescentes mexicanos.

\section{Método}

\section{Participantes}

En la fase 1 participaron tres traductoras certificadas, una de las autoras de la versión en inglés y el equipo de investigación del presente estudio. En la fase 2 se realizaron entrevistas cognitivas a una muestra no probabilística de 10 adolescentes, divididos en dos grupos por nivel educativo; los participantes del primer grupo eran estudiantes de una secundaria pública ( 2 mujeres y 3 hombres) con una edad comprendida entre los 12 y 14 años. El segundo grupo fue conformado por estudiantes de una preparatoria pública (3 mujeres y 2 hombres) con un rango de edad entre los $15 \mathrm{y}$ 18 años. En la fase 3 participó una muestra no probabilística integrada por 385 adolescentes entre los 11 y 19 años de edad, estudiantes de una secundaria y una preparatoria pública. Para el análisis factorial confirmatorio se consideró una muestra probabilística de 1608 adolescentes de 12 a 19 años, estudiantes de 3 secundarias y de 3 preparatorias de Ciudad Guzmán, Jalisco en México.

\section{Diseño}

El estudio se realizó en 3 fases. En la fase 1 se realizó la traducción y retro-traducción del ISSIQ-A y en la fase 2 la evaluación de la equivalencia conceptual mediante entrevistas cognitivas a adolescentes, mientras que en la fase 3 se evaluaron las propiedades psicométricas de la versión mexicana del ISSIQ-A.

\section{Instrumentos}

Para evaluar la validez convergente se utilizó el Cuestionario de Autolesionismo (Self-Harm Questionnaire, SHQ) de Ougrin \& Boege (2013) validado en México por GarcíaMijares et al. (2015) Su objetivo es detectar autolesión en adolescentes. Es un cuestionario de autoreporte de 15 ítems que posee confiabilidad (.96 alfa de Cronbach), validez y una sensibilidad para detectar autolesión de 97.96\%.

Para evaluar la validez divergente se aplicó el cuestionario KIDSCREEN-52 (Screening for and promotion of HealthRelated Quality of Life in Children and Adolescents). Fue elaborado por Ravens-Sieberer et al. (2008) y adaptado a la población mexicana por Hidalgo-Rasmussen, Rajmil \& Montaño (2014). Se utiliza en personas de 8 a 18 años de edad. La versión de 52 ítems mide 10 dimensiones de la calidad de vida relacionada con la salud: Bienestar Físico (5 ítems), Bienestar Psicológico (6 ítems), Estado de Ánimo (7 ítems), Autopercepción (5 ítems), Autonomía (5 ítems), Relación con los Padres y Vida Familiar (6 ítems), Recursos Económicos (3 ítems), Amigos y Apoyo Social (6 ítems), Entorno Escolar (6 ítems) y Aceptación Social (3 ítems). Se responde en escala Likert con cinco opciones de respuesta. A mayor puntaje mejor autopercepción. Posee validez de contenido y de constructo. La consistencia interna es alta en la mayoría de las dimensiones y aceptable en tres dimensiones (alfa de Cronbach .51 valor mínimo a .86 valor máximo).

\section{Procedimiento}

Para la fase 1 de adaptación lingüística se realizó la traducción-retrotraducción ya que se considera el método que garantiza mayor calidad en la traducción (Maneesriwongul \& Dixon, 2004). Durante el periodo de julio a agosto de 2016, dos traductoras certificadas, nativas de la lengua inglesa realizaron por separado la traducción del inglés al castellano. Ambas calificaron la dificultad de traducir cada uno de los ítems en un rango de 0 a 10 puntos, donde 0 fue menor dificultad. Posteriormente el equipo de investigación en conjunto con una de las autoras de la versión en inglés del ISSIQ-A revisaron cada uno de los ítems verificando la equivalencia semántica y de contenido para generar la primera versión del ISSIQ-A en castellano. Después una tercera traductora certificada, con el idioma castellano como lengua materna, realizó la retro-traducción al inglés y calificó la dificultad de cada ítem traducido. La versión retro-traducida fue comparada con la versión del IssiQ-A en inglés y la versión resultante fue aprobada por los autores de la versión en inglés.

En la fase 2 se realizaron entrevistas cognitivas a dos grupos de adolescentes estudiantes en septiembre de 2016. Se desarrollaron durante el horario escolar en las bibliotecas de las escuelas. Se solicitó a los participantes que contestaran el cuestionario y que indicaran en voz alta o por escrito cualquier duda o comentario que quisieran hacer; se enfatizó que no existían respuestas "correctas o incorrectas". Al terminar de contestar el cuestionario, un autor de este estudio registró el tiempo dedicado a la contestación y pidió a los participantes que evaluaran entre 0 y 10 la dificultad o el cansancio que experimentaron al contestarlo. Posteriormente se llevó a cabo una entrevista semi-estructurada para verificar que todos los ítems fueran comprendidos. Se evaluó la claridad de las instrucciones, la claridad de cada uno de los ítems, las categorías de respuesta, la sensibilidad a los temas tratados y los conocimientos o supuestos sobre la experiencia de autolesión no suicida. 
Se pidió a los participantes que leyeran cada uno de los aspectos evaluados y que explicaran con sus palabras lo que entendían. Cuando se generaron dudas o comentarios, se preguntó al resto de los participantes lo que entendían y se verificó si existía concordancia en el resto del grupo. El audio de la entrevista en estudiantes de preparatoria fue grabado. Los estudiantes de secundaria no autorizaron la grabación de audio, por lo que se tomaron apuntes en lápiz y papel. Las respuestas y comentarios realizados por los participantes fueron analizados por el equipo de investigación y discutidos con los autores de la versión original del ISSIQ-A para generar la versión final mexicana del ISSIQ-A.

En la fase 3 se aplicó la versión mexicana del ISSIQ-A para evaluar sus propiedades psicométricas. Se aplicó también el cuestionario de autolesionismo y el KIDSCREEN-52. Las aplicaciones se realizaron durante los meses de septiembre de 2016 y de febrero a marzo de 2017 a estudiantes dentro de su horario escolar. La reproducibilidad se valoró con datos de una submuestra $(n=332)$ proveniente de los 385 adolescentes a quienes se les aplicó el cuestionario por segunda ocasión con una diferencia de una semana entre cada aplicación.

\section{Consideraciones éticas}

El presente estudio fue evaluado y aprobado por el Comité de Bioética de la Universidad a cargo del estudio con el registro CB/008/2016. Se consideraron los principios bioéticos de la Declaración de Helsinki. En cada una de las fases del estudio se solicitó por escrito el consentimiento informado de los padres o tutores de adolescentes menores de edad y el asentimiento informado de todos los participantes, así como la autorización por escrito de las autoridades escolares. La información fue confidencial. El 15 de julio de 2016 se obtuvo la aprobación por escrito de los autores del ISSIQ-A para realizar la adaptación en población mexicana.

\section{Análisis estadísticos}

Los datos cualitativos obtenidos a partir del proceso de traducción-retrotraducción (fase 1) y de las entrevistas cognitivas a los participantes (fase 2) fueron analizados por los autores del presente estudio en conjunto con una de las autoras de la versión en inglés del ISSIQ-A para identificar dificultades en la comprensión de las instrucciones, la claridad de los ítems, las categorías de respuesta, la sensib ilidad a los temas tratados y los supuestos sobre la experiencia de autolesión no suicida. En la fase 3 se calculó el efecto piso techo; se consideró para piso la cantidad de participantes que contestaron en todos los ítems de un dominio la categoría nunca me ocurre y para techo se consideró la categoría me ocurre todo el tiempo, considerando como aceptable 15\% (Nunnally \& Bernstein, 1995). Se evaluó la fiabilidad del instrumento verificando la consistencia interna a partir del alfa de Cronbach para cada dimensión y para el cuestionario completo, siendo aceptable entre .70 y .95 (Terwee et al., 2007). La reproducibilidad se evaluó calculando kappa ponderada para cada dominio y para cada ítem, considerando la valoración de Landis \& Koch (1977) y un intervalo de confianza al $95 \%$ con el programa Stata SE versión 14. Además, se calculó el coeficiente de correlación de Spearman para analizar la validez convergente y la divergente con los criterios $r<0.30$ débil, $r=0.30-0.70$ moderada y $r>0.70$ asociación fuerte (Sánchez-Villegas, Martín-Calvo \& Martínez-González, 2014). Los datos se analizaron con el programa estadístico IBM SPSS versión 24. Para confirmar la estructura interna de cuatro factores y dos sub escalas del ISSIQ-A, se realizó un análisis factorial confirmatorio utilizando el modelo de máxima verosimilitud con los indicadores de bondad de ajuste, índice de ajuste comparativo (CFI 0.90 - 0.95 aceptable, $>0.95$ bueno; $\mathrm{Hu}$ \& Bentler, 1999), el error cuadrático medio de aproximación (RMSEA $<0.05$ buen ajuste, $0.05-0.08$ aceptable y $>0.08$ mediocre; Browne \& Cudeck, 1993), el índice de Tucker Lewis (TLI $\geq 0.90$; Lévy \& Varela, 2006), el CFI ajustado por parsimonia (PCFI > 0.06; Marôco, 2014) y chi cuadrado dividido por grados de libertad $\left(X^{2} / \mathrm{gl} \leq 5.0\right.$; Hair, Anderson, Tatham \& Black, 1999). Para el análisis factorial confirmatorio se utilizó el programa IBM SPSS AMOS versión 22.

\section{Resultados}

En la fase 1 la traducción al castellano fue considerada de baja dificultad de acuerdo a la evaluación de las dos traductoras. De los 56 ítems, las traductoras calificaron $\sin$ dificultad (0 puntos) a 43 ítems que representan el $76.7 \%$, 7 ítems (12.5\%) que tuvieron alguna dificultad (1 punto), 3 ítems (5.3\%) tuvieron 2 puntos de dificultad, 1 ítem (1.78\%) obtuvo 3 puntos de dificultad y solamente 2 ítems (3.5\%) obtuvieron 4 puntos de dificultad. A partir de la versión traducida acordada por los autores de este estudio, una de las autoras de la versión en inglés realizó 13 comentarios que fueron considerados para lograr la equivalencia semántica, incluyendo los ítems, instrucciones y opciones de respuesta. La retrotraducción al inglés fue considerada sin dificultad por la traductora. De los 56 ítems, calificó al 91\% de los ítems sin dificultad (0 puntos), 4 ítems (7\%) los calificó con alguna dificultad (1 punto) y 1 ítem (1.8\%) lo calificó con 2 puntos de dificultad. La versión retro-traducida fue 
Tabla 1. Comparación de la versión original del ISSIQ-A, con la versión retro-traducida y la versión mexicana final

\begin{tabular}{|c|c|c|}
\hline Versión original en inglés & Retrotraducción de la versión mexicana & Versión mexicana final \\
\hline $\begin{array}{l}\text { The following sentences depict the way } \\
\text { that youths feel from time to time. Please, } \\
\text { read each sentence carefully and check the } \\
\text { response that best express what happens } \\
\text { to you. There are no "right" or "wrong" } \\
\text { answers. }\end{array}$ & $\begin{array}{l}\text { The following sentences describe the way } \\
\text { sometimes young people feel. Please, read } \\
\text { each of the following sentences carefully } \\
\text { and choose the answer which best de- } \\
\text { scribes what happens to you. There are no } \\
\text { "correct" or "incorrect" answers. }\end{array}$ & $\begin{array}{l}\text { Los siguientes enunciados describen la } \\
\text { forma en la que los jóvenes se sienten a } \\
\text { veces. Por favor, lee cuidadosamente cada } \\
\text { uno de los enunciados y marca la respuesta } \\
\text { que mejor exprese lo que te ocurre. No hay } \\
\text { respuestas "correctas" o "incorrectas". }\end{array}$ \\
\hline 0. It never happens to me & 0 . It never happens & 0. Nunca me ocurre \\
\hline 1. It happens sometimes & 1. It sometimes happens & 1. Algunas veces me ocurre \\
\hline 2. It happens a lot & 2. It happens often & 2. Me ocurre mucho \\
\hline 3. It happens to me all the time & 3. It happens all the time & 3. Me ocurre todo el tiempo \\
\hline Other behaviors (please describe): & Other behaviors (please, describe them): & $\begin{array}{l}\text { Otros comportamientos (por favor descrí- } \\
\text { belos): }\end{array}$ \\
\hline $\begin{array}{l}\text { If you answered } 0 \text { to all previous questions, } \\
\text { please skip the section D. If you have any } \\
\text { answer with } 1 \text { or more, please continue to } \\
\text { the next section. }\end{array}$ & $\begin{array}{l}\text { If you answered } 0 \text { in all of the previous } \\
\text { answers, please don't answer the following } \\
\text { section and continue with section D. If you } \\
\text { answered any with } 1 \text { or more, please con- } \\
\text { tinue with the following section. }\end{array}$ & $\begin{array}{l}\text { Si respondiste } 0 \text { a todas las preguntas an- } \\
\text { teriores, por favor no contestes la siguiente } \\
\text { sección y continúa en la sección D. SI con- } \\
\text { testaste cualquiera con } 1 \text { o más, por favor } \\
\text { continúa en la siguiente sección. }\end{array}$ \\
\hline 4. I often lose my patience. & 4. I lose patience frequently. & 4. Con frecuencia pierdo la paciencia. \\
\hline $\begin{array}{l}\text { 13. I cut parts of my body on purpose } \\
\text { (With blades, scissors, knives, } x \text {-acto } \\
\text { knives, etc.). }\end{array}$ & $\begin{array}{l}\text { 13. I cut parts of my body on purpose (with } \\
\text { blades, scissors, knifes, cutter). }\end{array}$ & $\begin{array}{l}\text { 13. Corto partes de mi cuerpo a propósito } \\
\text { (con navajas, tijeras, cuchillos, cutter). }\end{array}$ \\
\hline $\begin{array}{l}\text { 22. I engage in promiscuous sexual } \\
\text { behavior (several partners, having } \\
\text { intercourse without protection, etc.). }\end{array}$ & $\begin{array}{l}\text { 22. I have high risk sexual behavior (with } \\
\text { multiple partners, having unprotected sex, } \\
\text { etc.). }\end{array}$ & $\begin{array}{l}\text { 22. Yo llevo a cabo comportamientos } \\
\text { sexuales de riesgo (con varias parejas, } \\
\text { teniendo relaciones sexuales sin } \\
\text { protección, etc.) }\end{array}$ \\
\hline 34. Hurting myself helps me to calm down. & 34. Hurting myself helps me calm down. & 34. El lastimarme me ayuda a calmarme. \\
\hline $\begin{array}{l}\text { 38. I hurt myself to draw other people's } \\
\text { attention. }\end{array}$ & $\begin{array}{l}\text { 38. I hurt myself to get attention from other } \\
\text { people. }\end{array}$ & $\begin{array}{l}\text { 38. Me lastimo para llamar la atención de } \\
\text { otras personas. }\end{array}$ \\
\hline $\begin{array}{l}\text { 55. Sometimes I think that I don't have a } \\
\text { future nor a way out. }\end{array}$ & $\begin{array}{l}\text { 55. Sometimes I don't think I have a future } \\
\text { or a way out. }\end{array}$ & $\begin{array}{l}\text { 55. Algunas veces pienso que no tengo un } \\
\text { futuro ni una salida. }\end{array}$ \\
\hline
\end{tabular}

aprobada sin modificaciones. En la tabla 1 se comparan algunos ítems de la versión original del ISSIQ-A, con la versión mexicana retrotraducida y la versión mexicana final.

Para la fase 2 la duración aproximada para contestar el cuestionario en los dos grupos $(n=10)$ fue de 10 minutos. Los participantes valoraron en un rango de 0 a 10 la percepción de dificultad o cansancio al contestar el cuestionario, otorgando puntuaciones de 1 a 3 , por lo que consideramos que el ISSIQ-A es adecuado para los adolescentes. En ambos grupos los participantes mencionaron que las instrucciones y opciones de respuesta fueron claras. En el grupo de 12 a 14 años, reportaron confusión respecto al término drogas blandas por lo que se modificó el ítem aclarando que se refiere a los derivados de marihuana. En sensibilidad, ambos grupos hicieron referencia a la deseabilidad social, considerando la posibilidad de que algunos adolescentes no reporten la autolesión "debido al miedo de que se enteren sus papás o por el posible acoso de los compañeros". Con respecto a los conocimientos sobre la experiencia de autolesión no suicida, en ambos grupos consideraron que "las conductas del cuestionario son comunes, sobre todo en los de primero o segundo año de secundaria [...] pero es raro que contesten con la verdad [...] a menos que sea anónimo". Después de considerar las entrevistas cognitivas, se realizaron modificaciones a seis palabras para lograr la equivalencia semántica.

En la fase 3 la muestra de 385 adolescentes consistió en 165 hombres $(42.90 \%)$ y 220 mujeres $(57.10 \%)$ con un promedio de edad de 13.82 años $(S D=1.4)$. La muestra para el análisis factorial confirmatorio fue de 1608 adolescentes, 
Tabla 2. Variables sociodemográficas de los adolescentes participantes en la fase 3

\begin{tabular}{|c|c|c|c|c|}
\hline \multirow{2}{*}{ Variables } & \multicolumn{2}{|c|}{$\begin{array}{l}\text { Muestra fase } 3 \\
\quad(n=385)\end{array}$} & \multicolumn{2}{|c|}{$\begin{array}{l}\text { Muestra para análisis factorial confirmatoric } \\
\qquad(n=1608)\end{array}$} \\
\hline & $n$ & $\%$ & $n$ & $\%$ \\
\hline \multicolumn{5}{|l|}{ Sexo } \\
\hline Hombre & 165 & 42.90 & 749 & 46.60 \\
\hline Mujer & 220 & 57.10 & 858 & 53.40 \\
\hline \multicolumn{5}{|l|}{ Edad } \\
\hline 11-14 años & 221 & 57.40 & 715 & 44.50 \\
\hline 15-19 años & 164 & 42.60 & 892 & 55.50 \\
\hline \multicolumn{5}{|l|}{ Nivel educativo } \\
\hline Secundaria & 203 & 52.70 & 795 & 49.50 \\
\hline Preparatoria & 182 & 47.30 & 812 & 50.50 \\
\hline \multicolumn{5}{|l|}{ Nivel socioeconómico* } \\
\hline Medio alto y alto & 83 & 21.60 & 393 & 24.50 \\
\hline Medio bajo & 205 & 53.20 & 805 & 50.10 \\
\hline Clase alta trabajadora & 70 & 18.20 & 309 & 19.20 \\
\hline Clase baja trabajadora & 11 & 2.90 & 82 & 5.10 \\
\hline
\end{tabular}

Nota: ${ }^{*}$ La cantidad de casos en nivel socioeconómico no coincide con el total de la muestra ya que algunos participantes desconocían la información.

Tabla 3. Fiabilidad, estabilidad y efecto piso techo de las dimensiones del ISSIQ-A en adolescentes mexicanos

\begin{tabular}{|c|c|c|c|c|c|c|}
\hline \multirow[t]{2}{*}{ Dimensiones } & $\alpha$ & $\begin{array}{l}\kappa \\
(\mathrm{IC} 95 \%)\end{array}$ & $\begin{array}{l}\text { Min } \kappa \\
(\mathrm{IC} 95 \%)^{\mathrm{a}}\end{array}$ & $\operatorname{Max} \kappa(\operatorname{IC~} 95 \%)^{b}$ & Piso & Techo \\
\hline & \multicolumn{6}{|c|}{$(n=332$, test-retest $)$} \\
\hline Impulsividad & .73 & $.44(.41-.46)$ & $.49(.43-.53)$ & $.63(.59-.71)$ & 5.2 & 0 \\
\hline Autolesión & .71 & $.47(.40-.51)$ & $.23(.01-.33)$ & $.68(.57-.81)$ & 56.1 & 0 \\
\hline Comportamientos de riesgo & .67 & $.39(.33-.49)$ & $.25(.12-.36)$ & $.73(.37-.83)$ & 82.0 & 0 \\
\hline Ideación Suicida & .81 & $.50(.47-.56)$ & $.55(.46-.59)$ & $.71(.65-.74)$ & 57.7 & 0.3 \\
\hline Reforzamiento automático & .94 & $55(.54-.63)$ & $.16(.05-.40)$ & $.67(.62-.79)$ & 54.8 & 0 \\
\hline Reforzamiento social & .86 & $.52(.50-.67)$ & $.21(.07-.32)$ & $.61(.44-.83)$ & 77.1 & 0 \\
\hline Puntuación total & .93 & $.50(.46-.58)$ & NA & NA & NA & NA \\
\hline
\end{tabular}

Nota: Se consideró tanto para piso como para techo la cantidad de participantes que contestaron en todos los ítems de un dominio la categoría más baja o más alta respectivamente. Piso = Nunca me ocurrió; Techo = Me ocurre todo el tiempo. En las celdas se muestran porcentajes; NA = No aplica; $\alpha=$ alfa de Cronbach; $\kappa=$ Kappa ponderado; IC 95\%, Intervalos de confianza al 95\%.

a = Se refiere a la Kappa Ponderada más baja de los ítems de la dimensión señalada; ${ }^{b}=$ Se refiere a la Kappa ponderada más alta de los ítems de la dimensión señalada. 
Mónica GuZmán-González, María Barraza, Francisca Bastías,

Tabla 4. Correlación de los items del ISSIQ-A con el SHQ y el KIDSCREEN-52 en estudiantes adolescentes mexicanos

\begin{tabular}{|c|c|c|c|c|c|c|c|c|c|c|c|}
\hline ISSIQ-A & SHQ & KS-BF & KS-BP & KS-EA & KS-AP & KS-AU & KS-PVF & KS-AAS & KS-EE & KS-AS & KS-RE \\
\hline Puntuación total & $.533 * * *$ & $-.297 * * *$ & $-.383 * * *$ & $-.464 * * *$ & $-.419 * * *$ & $-.345^{* * *}$ & $-.383 * * *$ & $-.271 * * *$ & $-.329 * * *$ & $-.224 * * *$ & $-274 * * *$ \\
\hline Impulsividad & $.321^{* * *}$ & $-.321^{* * *}$ & $-.341 * * *$ & $-.409 * * *$ & $-.383 * * *$ & $-.277 * * *$ & $-.340 * * *$ & $-.139 * *$ & $-.310 * * *$ & $-.275^{* * *}$ & $-.179 * *$ \\
\hline Autolesión & $.471^{* * *}$ & $-.297 * * *$ & $-.383 * * *$ & $-.464 * * *$ & $-.419 * * *$ & $-.345^{* * *}$ & $-.383 * * *$ & $-.271 * * *$ & $-.329 * * *$ & $-.224 * * *$ & $-.274 * * *$ \\
\hline $\begin{array}{l}\text { Comportamientos de } \\
\text { riesgo }\end{array}$ & $.196^{* * *}$ & -.08 & $-.111^{*}$ & -.073 & -.061 & -.066 & -.07 & -.019 & -.085 & -.077 & -.055 \\
\hline Ideación Suicida & $.586^{* * *}$ & $-.408^{* * *}$ & $-.479 * * *$ & $-.581 * * *$ & $-.497 * * *$ & $-.437 * * *$ & $-.472 * * *$ & $-.236^{* * *}$ & $-.323 * * *$ & $-.212 * * *$ & $-.383 * * *$ \\
\hline $\begin{array}{l}\text { Reforzamiento au- } \\
\text { tomático }\end{array}$ & $.451 * * *$ & $-.209 * * *$ & $-.329 * * *$ & $-.347 * * *$ & $-.286^{* * *}$ & $-.228 * * *$ & $-.373^{* * *}$ & $-.202 * * *$ & $-.310^{* * *}$ & $-.212 * * *$ & $-.364 * * *$ \\
\hline Reforzamiento social & $.419^{* * *}$ & $-.249 * * *$ & $-.293 * * *$ & $-.319 * * *$ & $-.296 * * *$ & $-.240 * * *$ & $-.298 * * *$ & $-.168 * *$ & $-.199 * * *$ & $-.206 * * *$ & $-.349^{* * *}$ \\
\hline
\end{tabular}

Nota: Se utilizó correlación de Spearman; KS = dominio de KIDSCREEN-52; KS-BF = Bienestar Físico, KS-BP=Bienestar Psicológico, KS-EA = Estado de Ánimo, KS-AP = Autopercepción, KS-AU = Autonomía, KS-PVF = Padres y Vida Familiar, KS-AAS = Amigos y Apoyo Social, KS-EE = Entorno Escolar, KS-AS = Aceptación Social, KS-RE = Recursos Económicos.

$* \mathrm{p}<.05 ; * * \mathrm{p}<.01 ; * * \mathrm{p}<.001$

749 hombres $(46.60 \%)$ y 858 mujeres $(53.40 \%)$ con una edad media de 14.77 años $(S D=1.80$; tabla 2$)$. La fiabilidad fue adecuada en el cuestionario completo y en la mayoría de las dimensiones. El coeficiente alfa de Cronbach del cuestionario fue de .93 y por dimensiones en un rango de $\alpha_{\min }=.67 \mathrm{a}$ $\alpha_{\max }=.94$. La reproducibilidad fue moderada tanto para el cuestionario completo ( $\kappa=.50$, IC $95 \% .46-.58)$, como para la mayoría de las dimensiones, excepto la dimensión de Comportamientos de Riesgo que fue baja ( $\kappa=.39$, IC $95 \%$ .33-.49). Los porcentajes de piso fueron mayores al $15 \%$ en la mayoría de los dominios, con excepción del dominio Impulsividad (tabla 3).

Tal como se esperaba, se encontraron correlaciones positivas estadísticamente significativas $(p<.001)$ entre el ISSIQ-A y el cuestionario de autolesionismo (SHQ). La mayoría de las correlaciones fueron moderadas $(r=.32-.58)$ a excepción del dominio Comportamientos de Riesgo que fue débil $(r=.19)$. Las correlaciones del IssiQ-A con el cuestionario KIDSCREEN-52 fueron negativas, estadísticamente significativas $(p<.001)$ y de débiles a moderadas $(r=-.11$ a -.58) para todas las dimensiones, excepto Comportamientos de Riesgo que sólo fue significativa $(p<.05)$ para el dominio Bienestar Psicológico con una correlación débil $(r=-.11$; tabla 4$)$.

Los indicadores de bondad de ajuste del modelo con cuatro factores fueron aceptables: CFI $=0.903$, RMSEA $=.058$,

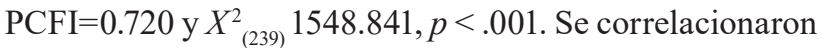
los errores de los ítems considerando los índices de modificación mayores, siempre y cuando pertenecieran a la misma dimensión. Todos los ítems obtuvieron cargas factoriales estandarizadas mayores a .30 (tabla 5). Autolesión No Suicida obtuvo correlaciones moderadas con Ideación Suicida $r=0.54$, Impulsividad $r=0.38$ y Comportamientos de Riesgo $r=0.57$. El modelo con las dos subescalas también obtuvo aceptables indicadores de bondad de ajuste: $\mathrm{CFI}=0.919$, RMSEA $=.070$, TLI $=0.900$, PCFI $=0.749$ y $X^{2}{ }_{(379)} 3394.077$, $p<.001$. Se correlacionaron los errores de los ítems con mayor índice de modificación que pertenecieran a la misma dimensión. Las cargas factoriales estandarizadas también fueron superiores a .30 (tabla 5). Se obtuvo una fuerte correlación entre la subescala de Reforzamiento Automático y la de Reforzamiento Social $(r=.91)$.

Tabla 5. Cargas factoriales estandarizadas de los items para cada dimensión del ISSIQ-A en adolescentes mexicanos

\begin{tabular}{lcc}
\hline Dimensiones & $\begin{array}{c}\text { Cantidad } \\
\text { de ítems }\end{array}$ & $\begin{array}{c}\text { Carga factorial } \\
\text { estandarizada }\end{array}$ \\
\hline Impulsividad & 8 & $.31-.70$ \\
Autolesión & 8 & $.53-.73$ \\
Comportamientos de riesgo & 6 & $.47-.79$ \\
Ideación Suicida & 3 & $.78-.82$ \\
Reforzamiento automático & 24 & $.46-.84$ \\
Reforzamiento social & 7 & $.52-.77$ \\
\hline
\end{tabular}

Nota: Se consideró adecuada una carga factorial mayor a .30 para cada ítem.

\section{Discusión}

El objetivo de este estudio fue adaptar transculturalmente el instrumento ISSIQ-A que evalúa autolesión no suicida y variables relacionadas en una población de estudiantes adolescentes mexicanos entre los 11 y 19 años. En la fase 1 se desarrolló el proceso de adaptación lingüística que 
permitió generar la primera versión mexicana del ISSIQ-A utilizado en la fase 2 de entrevistas cognitivas a partir de las cuales se confirmó que el ISSIQ-A es adecuado para su uso con adolescentes y que la versión mexicana del IssiQ-A es equivalente con la versión original en inglés. El tiempo, la dificultad y el cansancio al contestarlo fue poco. No se presentaron dificultades para comprender las instrucciones y opciones de respuesta. Un aspecto importante a considerar es la tendencia a la deseabilidad social que pudiera subestimar la presencia de autolesión no suicida pues, aunque en ambos grupos de participantes se hizo referencia a que las conductas de autolesión no suicida son comunes entre los pares, el estigma y/o el miedo a la reacción de los padres pudiera ser un factor importante por el cual se nieguen a reportar por escrito estas conductas, por lo que se recomienda garantizar el anonimato y la confidencialidad a los participantes.

En la fase 3 se reportó alta fiabilidad de la versión mexicana del IssiQ-A, similar a la primera versión en inglés. El coeficiente alfa de Cronbach fue alto en el instrumento completo como en la mayoría de las dimensiones. Únicamente en la dimensión de Comportamientos de Riesgo la consistencia interna y la reproducibilidad fueron bajas, mientras que en el resto de las dimensiones fue moderada. Probablemente esta baja consistencia y reproducibilidad se debe a que el instrumento no especifica cuál es la cantidad de alcohol considerada como abuso, dejando al criterio de los participantes lo que pueden entender por ese término. Ante tal situación los estudiantes podrían estar utilizando diferentes criterios en la primera y en la segunda aplicación. El porcentaje alto que se obtuvo en el efecto piso para la mayoría de las dimensiones es congruente con lo esperado en una población no clínica, ya que las variables evaluadas presentan menor prevalencia en la población general (Briere \& Gil, 1998; Dougherty et al., 2009). Con respecto a la validez de constructo, se comprobó la estructura de cuatro factores y dos subescalas propuesta en la primera versión en inglés del ISSIQ-A con indicadores de ajuste aceptables para la población mexicana, con pesos factoriales mayores a .30 en todos los ítems y correlaciones moderadas y fuertes entre las dimensiones del instrumento. Los resultados del análisis de validez convergente y divergente aportan evidencia de la validez del ISSIQ-A en población mexicana. Cabe mencionar que a pesar de que la primera versión del ISSIQ-A en inglés fue desarrollada para participantes de 14 a 21 años, los resultados de nuestro estudio muestran que es adecuada para adolescentes desde los 11 años de edad y hasta los 19 años.

Por otra parte, los resultados indican que el cuestionario podría ser útil tanto para identificar la presencia de conductas de NSSI, como para la evaluación de variables asociadas durante el tratamiento y/o seguimiento en adolescentes en los que ya han sido identificadas estas conductas, tales como el tipo de Reforzamiento, la Impulsividad, Comportamientos de Riesgo e Ideación Suicida. Sin embargo, es importante realizar otros análisis para verificar su adecuado funcionamiento en población clínica.

Como limitaciones de este estudio podemos mencionar que las muestras con las que se trabajó fueron de estudiantes, por lo que se recomienda realizar estudios para verificar sus propiedades psicométricas en otro tipo de población. Además, los estudiantes seleccionados pertenecían a una misma ciudad por lo que las variaciones culturales al interior de la república mexicana podrían no ser reflejadas; sin embargo, aun cuando en México existen importantes diferencias étnicas en poblaciones particulares, tales como grupos indígenas, en general los estudiantes que participan dentro del Sistema Educativo Nacional tienen una gran semejanza, por lo que consideramos que este instrumento podría ser útil en todo contexto escolar mexicano donde se hable español.

En este estudio se reporta el proceso de adaptación transcultural del cuestionario ISSIQ-A, mostrando evidencia de la equivalencia conceptual, semántica y de constructo de la versión mexicana con la versión original en inglés. Considerando su adecuada fiabilidad y validez, la versión mexicana del IsSIQ-A resulta útil para evaluar la conducta de autolesión no suicida y variables relacionadas en adolescentes mexicanos.

\section{Referencias}

Albores-Gallo, L., Méndez-Santos, J.L., García-Luna, A.X., Delgadillo-González, Y., Chávez-Flores, C.I., \& Martínez, O.L. (2014). Autolesiones sin intención suicida en una muestra de niños y adolescentes de la ciudad de México. Actas Españolas de Psiquiatría, 42(4), 159-68. Recuperado de https://www.actaspsiquiatria.es/repositorio/16/90/ ESP/16-90-ESP-159-168-346514.pdf

Briere, J., \& Gil, E. (1998). Self-mutilation in clinical and general population samples: Prevalence, correlates, and functions. American Journal of Orthopsychiatry, 68(4), 609-620. https://doi.org/10.1037/h0080369

Browne, M. W., \& Cudeck, R. (1993). Alternative ways of assessing model fit. En: Bollen, K. A. y Long, J. S. (Eds.), Testing structural equation models (136-162). Beverly Hills, CA: Sage.

Calvete, E., Orue, I., Aizpuru, L., \& Brotherton, H. (2015). Prevalence and functions of non-suicidal self-injury in Spanish adolescents. Psicothema, 27, 223-228. doi: 10.7334/psicothema2014.262

Carvalho, C.B., Nunes, C., Castilho, P., da Motta, C., Caldeira, S., \& Pinto-Gouveia, J. (2015). Mapping non-suicidal self-injury in adolescence: Development and confirmatory factor analysis of the impulse, self-harm and suicide ideation questionnaire for adolescents (ISSIQ-A). Psychiatry Research, 227 (2-3), 238-45. doi: 10.1016/j.psychres.2015.01.031 
Castro, S. E., Benjet, C., Juárez, G. F., Jurado, C. S., Lucio, G.M., \& Valencia, C. A. (2016). Adaptación y propiedades psicométricas del Inventory of Statements About Self-injury en estudiantes mexicanos. Acta de investigación psicológica, 6, 2544-2551. http://dx.doi. org/10.1016/j.aipprr.2016.08.004

Dougherty, D. M., Mathias, C. W., Marsh-Richard, D. M., Prevette, K. N., Dawes, M. A., Hatzis, E. S., .. \& Maziade, M. (2009). Impulsivity and clinical symptoms among adolescents with non-suicidal self-injury with or without attempted suicide. Psychiatry Research, 169(1), 22-7. https://doi.org/10.1016/j.psychres.2008.06.011

Duggan, J.M., \& Heath, N.L. (2013). Co-occurring health-risk behaviors of non-suicidal self-injury and eating disorders. En: Claes, L. y Muehlenkamp, J.J. Non-Suicidal Self-Injury in Eating Disorders: Advancements in Etiology and Treatment. (217-36). Berlin Heidelberg: Springer Science y Business Media.

García-Mijares, J.F., Alejo-Galarza, G.J., Mayorga-Colunga, S.R., Guerrero-Herrera, L.F., \& Ramírez-Garcíaluna, J.L. (2015). Validación al español del Self-Harm Questionnaire para detección de autolesionismo en adolescentes. Salud Mental, 38(4), 287-92. http://dx.doi.org/10.17711/ SM.0185-3325.2015.039

Hair, J., Anderson, R., Tatham, R., \& Black, W. (1999). Análisis Multivariante. (5. Ed.). Madrid: Prentice Hall.

Hamza, C.A., Willoughby, T., Heffer, T. (2015). Impulsivity and non-suicidal self-injury: A review and meta-analysis. Clinical Psychology Review, 38, 13-24. doi: 10.1016/j.cpr.2015.02.010

Hawton, K., \& Van Heeringen, K. (2008). The International Handbook of Suicide and Attempted Suicide. England: Wiley.

Hidalgo-Rasmussen, C. A., Rajmil, L., \& Montaño, R. (2014). Adaptación transcultural del cuestionario KIDSCREEN para medir calidad de vida relacionada con la salud en población mexicana de 8 a 18 años. Ciéncia y Saude Coletiva, 19(7), 2215-2224. https://doi.org/10.1590/141381232014197.09682013

Hu, L. \& Bentler, P. M. (1999). Cutoff criteria for fit indexes in covariance structure analysis: conventional criteria versus new alternatives. Structural Equation Modeling: A multidisciplinary Journal, 6(1), 1-55. http://dx.doi.org/10.1080/10705519909540118

Klonsky, E.D. (2011). Non-suicidal self-injury in United States adults: prevalence, sociodemographics, topography and functions. Psychological Medicine, 41(9), 1981-6. doi: 10.1017/S0033291710002497

Landis, J.R., \& Koch, G.G. (1977). The Measurement of Observer Agreement for Categorical Data. Biometrics, 33(1), 159-74.

Lévy, J. P., \& Varela, J. (2006). Modelación con estructuras de covarianzas en ciencias sociales. Madrid: Netbiblo.

Manca, M., Presaghi, F., \& Cerutti, R. (2014). Clinical specificity of acute versus chronic self-injury: Measurement and evaluation of repetitive non-suicidal self-injury. Psychiatry Research, 215(1), 111-119. https:// doi.org/10.1016/j.psychres.2013.10.010

Maneesriwongul, W., \& Dixon, J.K. (2004). Instrument translation process: a method review. Journal of Advanced Nursing, 48(2), 175-86. doi: 10.1111/j.1365-2648.2004.03185.x

Marôco, João. (2014). Análise de Equações Estruturais. Fundamentos teóricos, Software e Aplicações. Pêro Pinheiro: ReportNumber.
Mayer, V.P., Morales, G.N., Figueroa, G.V., \& Ulloa, F.R. (2016). Adolescentes con autolesiones e ideación suicida: un grupo con mayor comorbilidad y adversidad psicosocial. Salud Pública de México, 58, 335-6. doi: 10.21149/spm.v58i3.7893

Muehlenkamp, J.J, Claes, L., Havertape, L., \& Plener, P.L. (2012). International prevalence of adolescent non-suicidal self-injury and deliberate self-harm. Child and Adolescent Psychiatry and Mental Health, 6(10), 2-9. doi: 10.1186/1753-2000-6-10

Muehlenkamp, J.J., \& Gutiérrez, P.M. (2007). Risk for suicide attempts among adolescents who engage in non-suicidal self-injury. Archives of Suicide Research, 11(1), 69-82. doi: 10.1080/13811110600992902

Nock, M.K. (2010). Self-Injury. Annual Review of Clinical Psychology, 6(1), 339-63. doi:10.1146/annurev.clinpsy.121208.131258

Nock, M.K., \& Prinstein, M.J. (2004). A Functional Approach to the Assessment of Self-Mutilative Behavior. Journal of Consulting and Clinical Psychology, 72(5), 885-90. doi: 10.1037/0022-006X.72.5.885

Nunnally, J., \& Bernstein, I. (1995). Teoría psicométrica. (3. Ed). México DF: McGraw-Hill.

Ougrin, D., \& Boege, I. (2013). Brief report: The Self Harm Questionnaire: a new tool designed to improve identification of self-harm in adolescents. Journal of Adolescent, 36(1), 221-5. doi:10.1016/j. adolescence.2012.09.006

Ravens-Sieberer, U., Gosch A., Rajmil L., Erhart M., Bruil J., Power M., Duer W., Auquier P., Cloetta B., Czemy L., Mazur J., Czimbalmos A., Tountas Y., Hagquist C., K. J. . K. G. (2008). The KIDSCREEN-52 quality of life measure for children and adolescents: psychometric results from cross-cultural survey in 13 european countries. Value Health, 11(4), 645-658. doi: 10.1111/j.1524-4733.2007.00291.x

Sánchez-Villegas, A., Martín-Calvo, N, \& Martínez-González, M.A. (2014). Correlación y regresión lineal simple. En M. A. MartínezGonzález, A. Sánchez-Villegas, E. Toledo, y J. Faulin Fajardo. (Eds.). Bioestadística amigable (pp. 269-326). Barcelona: Elsevier.

Saraff, P.D., Pepper, C.M. (2014). Functions, lifetime frequency, and variety of methods of non-suicidal self-injury among college students. Psychiatry Research, 219(2), 298-304. doi: 10.1016/j.psychres.2014.05.044

Terwee, C.B., Bot, S., Van Der Windt, D.A., \& Dekker J. (2007). Quality criteria were proposed for measurement properties of health status questionnaires. Journal of Clinical Epidemiology, 60(1), 34-42. doi: 10.1016/j.jclinepi.2006.03.012

Tørmoen, A.J., Rossow, I., Larsson, B., \& Mehlum, L. (2013). Nonsuicidal self-harm and suicide attempts in adolescents: differences in kind or in degree? Social Psychiatry and Psychiatric Epidemiology, 48(9), 1447-55. doi:10.1007/s00127-012-0646-y

Vrouva, I., Fonagy, P., Fearon, P. R. M., \& Roussow, T. (2010). The risk-taking and self-harm inventory for adolescents: Development and psychometric evaluation. Psychological Assessment, 22(4), 852-865. https://doi.org/10.1037/a0020583

Washburn, J. J., Juzwin, K. R., Styer, D. M., \& Aldridge, D. (2010). Measuring the urge to self-injure: Preliminary data from a clinical sample. Psychiatry Research, 178(3), 540-544. https://doi.org/10.1016/j. psychres.2010.05.018 
